\title{
Comprehensive Evaluation and Analysis of the Salinity Stress Response Mechanisms Based on Transcriptome and Metabolome of Staphylococcus Aureus
}

\author{
Ying Feng \\ Ningbo University \\ Dizhou Gu \\ Tonghua Normal University \\ Ziyan Wang \\ Ningbo University \\ Chenyang Lu \\ Ningbo University \\ Jingfeng Fan \\ National Marine Environmental Monitoring Center \\ Jun Zhou \\ Ningbo University \\ Rixin Wang \\ Ningbo University \\ Xiurong Su ( $\nabla$ suxiurong_public@163.com ) \\ Ningbo University https://orcid.org/0000-0003-2336-191X
}

\section{Research Article}

Keywords: Staphylococcus aureus, salinity stress, transcriptome, metabolome

Posted Date: June 30th, 2021

DOI: https://doi.org/10.21203/rs.3.rs-641989/v1

License: (c) (i) This work is licensed under a Creative Commons Attribution 4.0 International License. Read Full License

Version of Record: A version of this preprint was published at Archives of Microbiology on December 18th, 2021. See the published version at https://doi.org/10.1007/s00203-021-02624-9. 


\section{Abstract}

Staphylococcus aureus possesses an extraordinary ability to deal with a wide range of osmotic pressure. To performed transcriptomic and metabolomic analyses on the potential mechanism of gradient salinity stress adaptation in S. aureus ZS01. The results revealed that CPS biosynthetic protein genes were candidate target genes for directly regulating the phenotypic changes of biofilm. Inositol phosphate metabolism was downregulated to reduce the conversion of functional molecules. Gluconeogenesis pathway was downregulated to reduce the production of endogenous glucose. Pyruvate metabolism pathway was upregulated to promote the accumulation of succinate. TCA cycle metabolism pathway was downregulated to reduce unnecessary energy loss. These self-protection mechanisms can protect cells from hypertonic environments, and help them focus on survival. In addition, we identified 10 hub genes. The findings will aid in the prevention and treatment strategies of $S$. aureus infections.

\section{Background}

Staphylococcus aureus is an important opportunistic pathogen, which is highly resistant to osmotic stress (Schuster et al., 2016). S. aureus strains can generate a large scale of extracellular toxic proteins while growing or occurring in food, resulting in the outbreaks of staphylococcal food poisoning (SFP) in humans and animals (Alibayov et al., 2014). SFP is a worldwide public health problem, mainly caused by its enterotoxin, hemolysin, and leukotoxin (Loir et al., 2003). Besides, S. aureus can adapt and survive in harsh environments, such as drought, cold, and salinity stress (Argudín et al., 2010; Sergelidis et al., 2015). Moreover, it has a strong tolerance for many common bacteriostatic methods, and SFP accounts for a high proportion of bacterial food poisoning (Fischer et al., 2009). Therefore, research on $S$. aureus has become an important research area in the field of food safety (Xu et al., 2019).

S. aureus is considered the main pathogenic bacteria in aquatic product processing. Traditional aquatic product processing uses high-salted pickling methods to inhibit the growth of microorganisms in aquatic products, and thus prevent corruption in long-term preservation (Fuentes et al., 2010). However, the existing reports are seriously and increasingly concerned about the prevention and treatment of drugresistant bacteria, which distracts attention from exploring the fundamental mechanism of conferring tolerance to salinity fluctuations (Wong et al., 2019; Ai et al., 2020). Therefore, the molecular mechanism of $S$. aureus for the tolerance of extremely high salt stress was studied, and its tolerance to high salt stress was weakened in a targeted way to enhance the inhibitory effect in aquatic products. The control methods of $S$. aureus are important research directions in the field of aquatic product processing, and have practical significance for the diagnosis of diseases (Price-Whelan et al., 2013). Therefore, we studied the molecular mechanism underlying the response of $S$. aureus ZS01 to extremely high salt stress. A targeted weakening of its tolerance to high salt stress can be developed as a new therapy other than antibiotics.

Generally, osmotic stress often has a great effect on the structure, chemistry, and physiology of a bacterial cell. According to previous studies on bacteria response to salinity stress, biofilm assays 
showed a positive correlation between biofilm formation and increased concentration of $\mathrm{NaCl}$ (Beckingsale and Thomas, 2008). Islam et al. demonstrated that the addition of $\mathrm{NaCl}$ increased the production of polysaccharide intercellular adhesin (PIA) to induce changes in biofilm (Islam N 2015).

There have been several studies on osmoregulation in S. aureus (Graham and Wilkinson, 1992; Cebrián et al., 2015), and choline, glycine betaine, L-proline, and taurine have demonstrated osmotic protective effect (Vijaranakul et al., 1995). The accumulation of permeating agents can effectively help bacteria to survive in a hyperosmolar environment. For example, Listeria monocytogenes respond to a hyperosmolar environment by transporting proteins to absorb osmotic protectants; Bacillus subtilis osmotically regulates by the synthesis of betaine (Boch et al., 1994; Angelidis and Smith, 2003). It has been reported that Enterococcus faecalis can reduce the metabolism of carbohydrates and amino acids, and increase the synthesis of nucleotide to adapt alkaline stress (Ran et al., 2015b). There are many reports about the cellular and signaling molecular responses of bacteria to osmotic stress. Escherichia coli increased the expression of channel proteins through the EnvZ/OmpR two-component regulatory system to increase tolerance to hyperosmotic environments (Oshima et al., 2002). In Streptococcus mutans, ATP-binding cassette $(A B C)$ transporters act as sugar metabolism transporters to resist environmental stress (Nagayama et al., 2014).

In recent years, omics research has predominantly been used to explore these biological phenomena. It mainly relies on the rapid development of high-throughput sequencing technology (Xin et al., 2019). The application of these platforms makes outstanding contributions to in-depth research on the occurrence of the mechanisms. With transcriptomics data as the research background, Guan et al. evaluated the role of the afap 1 in oxidative stress and aflatoxin synthesis (Guan et al., 2019). Kim et al. explained the thermotolerant mechanism of E. coli by using transcriptome and metabolome analyses (Kim et al., 2020). In this study, transcript and metabolite datasets have been combined to reveal the response mechanism via correlation and cluster analyses, and further manifested as the connection networks between genes and metabolites. Salt-tolerant strains can evolve specific mechanisms in response to salt stress and changes. This mechanism was studied in our previous report, but it focused on the influence of DEPs at the proteomics level (Ming et al., 2019). Herein, an integrated analysis revealed more insights into the salinity stress-responsive genes, DMs, and pathway interactions than separate analysis. The screening of possible key genes and metabolites provides an opportunity for us to understand how S. aureus ZS01 responds to salt stress, helps us to prevent SFB infections, and develops new therapies other than antibiotics.

\section{Materials And Methods}

\section{Bacterial strain and growth conditions}

S. aureus ZS01 was separated from pickled aquatic products in Zhoushan City. Bacterial strain and growth conditions were determined using the method previously described by Ming et al (Ming et al., 2019). Briefly, S. aureus ZS01 was incubated for 48 hours in a broth medium supplemented with $0 \%, 10 \%$ and $20 \% \mathrm{NaCl}$, respectively. 


\section{Scanning electron microscope (SEM) analysis of morphological observation}

Take $1.5 \mathrm{~mL}$ of bacteria liquid and centrifuge at $8000 \times \mathrm{g}$ for $4 \mathrm{~min}$, discard the supernatant. The cells were processed for SEM according to the method described by Kong et al. (Kong et al., 2018). The samples were gold-coated with multifunctional sample surface treatment machine (Zhong et al., China). A Hitachi S3000N was used for the SEM image capture. The magnifications used were $\times 10000$.

\section{Biofilm analysis}

Appropriate dilutions were made to obtain a concentration of approximately $5 \times 10^{6} \mathrm{CFU} / \mathrm{mL}$. The solution was centrifuged at $8000 \times \mathrm{g}$ for 2 min to collect the cells. Biofilm was stained with FITC-ConA $\left(4^{\circ} \mathrm{C}, 30 \mathrm{~min}\right)$ and $\mathrm{PI}\left(4^{\circ} \mathrm{C}, 15 \mathrm{~min}\right)$, then imaged. Biofilm analysis was performed according to the method described by Rodriguez-Melcon et al. (Rodriguez-Melcon et al., 2019). A Zeiss LSM880 confocal laser scanning microscope (Zeiss LSM880, Germany) was used for the confocal laser scanning microscopy (CLSM) image capture. The Zen black 2.1 software is used to perform image analysis, biofilm thickness measure and export.

\section{Total RNA isolation and transcriptome analysis}

Total RNA was extracted using a commercial RNA purification kit (Invitrogen, California, USA), purity and quantity were analyzed using the Agilent2100 Bioanalyzer, and rRNA was removed using the Ribo-Zero Magnetic kit (G+/G-Bacteria) (Epicentre, Wisconsin, USA) according to the manufacturer's instruction. Sequencing was carried out on the Illumina Hiseq 4000 platform for $2 \times 100$ bp/300 bp (Majorbio BioPharm Technology Co., Ltd., Shanghai, China) (Ran et al., 2015a).

The raw sequence data (raw reads) were filtered with the Seq Prep and Sickle software to obtain clean data. The Q20, Q30, GC-content, and sequence duplication levels of the clean data were calculated (Zhang et al., 2017). High quality reads from each sample were aligned to the reference genome $S$. aureus ATCC 27217 (https://www.ebi.ac.uk/ena/data/view/GCA_000597965) using the Bowtie software (Zhao et al., 2018). Fragments per kilobase of transcript per million fragments represented the expression values of predicted $S$. aureus transcripts (Cole et al., 2010). An adjusted FDR $\leq 0.05$ and $\left|\log _{2} F C\right| \geq 1$ were regarded as significant differentially expressed genes (DEGs). Furthermore, enrichment analysis of the DEGs was analyzed via Gene Ontology (GO) enrichment analysis and the Kyoto Encyclopedia of Genes and Genomes (KEGG) pathway analysis to obtain a detailed description. Differences with a $p$-value of $\leq$ 0.05 were used as a threshold to determine the significant enrichment.

\section{Quantitative real-time PCR (qRT-PCR)}

Ten DEGs were selected for qRT-PCR to validate our Illumina sequencing data. Total RNA was extracted using the commercial TransZol Up Plus RNA Kit (TRAN, Beijing, China) according to the manufacturer's instruction. Reverse transcription was performed using the Prime Script ${ }^{\text {TM }}$ RT reagent kit (TRAN, Beijing, China). The primers were designed with the Primer Premier 5.0 software. The sequences of the primer 
pairs are listed in Table S1. Rotor-Gene 6000 realtime PCR machine (Corbett, Australia) and SYBR ${ }^{\circledR}$ Premix $\mathrm{Ex} \mathrm{Taq}^{\mathrm{TM}}$ II were used for qRT-PCR analysis. The relative gene expression was normalized internally to $16 \mathrm{~s}$ rDNA level and quantified according to the $2^{-\Delta \Delta C t}$ method.

\section{Metabolite analysis}

The extraction and derivatization of metabolites were performed as previously reported (Ming et al., 2018). Then gas chromatography-mass spectrometry (GC-MS) was used to analyze the metabolites. The column temperature programming was as follows: $90^{\circ} \mathrm{C}$ held for $3 \mathrm{~min}$; from $90^{\circ} \mathrm{C}$ to $160{ }^{\circ} \mathrm{C}$ at 3 ${ }^{\circ} \mathrm{C} / \mathrm{min}$, held for $0 \mathrm{~min}$; from $160{ }^{\circ} \mathrm{C}$ to $220^{\circ} \mathrm{C}$ at $2{ }^{\circ} \mathrm{C} / \mathrm{min}$, held for $1 \mathrm{~min}$; from $220^{\circ} \mathrm{C}$ to $290{ }^{\circ} \mathrm{C}$ at 10 ${ }^{\circ} \mathrm{C} / \mathrm{min}$, held for $0 \mathrm{~min}$. Principal component analysis (PCA) was performed using the SIMCA-P+ver14 software (Hashim et al., 2014). Pathway analysis was performed with MetaboAnalyst 4.0 (Chong et al., 2019).

\section{Integrated transcriptomeand metabolome analyses}

Spearman correlation test was used to analyze the correlation between candidate gene expression and discriminant metabolite content. Discriminant metabolites contain aminobutanoic acid, glycolic acid, Derythrofuranose, sebacic acid, xylitol, D-threitol, n-hexadecanoic acid, myo-Inositol, heptacosane, undecanedioic acid, L-proline, phosphoric acid, succinate, D-arabinose, and D-mannitol.

\section{Results}

\section{High concentrations of $\mathrm{NaCl}$ affect morphology}

In the control group, the morphology of $S$. aureus ZS01 was spherical, the surface of cell was smooth without damage or wrinkles, and the size was relatively neat (Fig. 1A). With the increase of salt concentration, there was no significant change in cell morphology in the $10 \% \mathrm{NaCl}$ treatment group, but the number of cells was significantly lower than that in the control group (Fig. 1B). In the $20 \% \mathrm{NaCl}$ treatment group, the cell membrane ruptured obviously, the cell contents overflowed, and some cells showed shrinkage (Fig. 1C).

From the above results, it can be seen that the morphological changes of $S$. aureus ZS01 in the $20 \% \mathrm{NaCl}$ treatment group are more obvious than in the $10 \% \mathrm{NaCl}$ treatment group. High salinity stress can change the permeability of cell membrane and rupture the cell membrane, leading to cell death.

\section{High concentrations of $\mathrm{NaCl}$ affect biofilm formation}

The distribution of the bacteria and extracellular polymeric substances (EPS) was observed from 3D views of CLSM images (Fig. 2A, 2B, and 2C). FITC-ConA can bind to cell wall polysaccharides to emit green fluorescence, and PI can penetrate bacterial cells and bind to DNA to emit red light. EPS as the main component of biofilm emits green fluorescence; bacterial DNA emits red fluorescence. With the increase of $\mathrm{NaCl}$ concentration, the thickness of biofilms increased and then decreased, and the 
difference between adjacent groups was significant (Fig. 2D). The results show that in the concentration range of less than $10 \% \mathrm{NaCl}$, high concentration of $\mathrm{NaCl}$ contributes to the formation of biofilms. When the concentration of $\mathrm{NaCl}$ is higher than $10 \%, \mathrm{NaCl}$ has an inhibitory effect on the formation of biofilm.

\section{Transcriptomic profiling through RNA-Seq}

Compared with the control group, 248 DEGs (121 upregulated and 127 downregulated) and 891 DEGs (365 upregulated and 526 downregulated) were identified in the $10 \%$ and $20 \% \mathrm{NaCl}$ groups, respectively (Fig. 3A and Fig. 3B). Compared with the 10\% NaCl group, 1063 DEGs (399 upregulated and 664 downregulated) were identified in the $20 \% \mathrm{NaCl}$ group (Fig. 3C). Furthermore, the number of downregulated genes was higher than that of the upregulated genes in the three groups. Comparing Fig. $3 \mathrm{~A}$ and Fig. $3 \mathrm{~B}$, it was found that as the salt concentration increased, more genes were mobilized to participate in the process of high salt stress.

Hierarchical cluster analysis of the DEGs was conducted using the Heml 1.0 software (Deng et al., 2014). Four expression change patterns were displayed among these DEGs with increasing concentrations of $\mathrm{NaCl}$ (Fig. 4). The four patterns are Pattern I (increase/increase); Pattern II (increase/decrease); Pattern III (decrease/decrease) and Pattern IV (decrease/increase) (Table S2). The numbers of DEGs that showed each pattern were $2,80,43$, and 19 , respectively. As a result, these DEGs can be considered as the candidate target genes for the direct regulation of salt stress. In addition, cluster analysis displayed that these genes affected by salt stress were abundant in some pathways related to membrane transport, redox process, metabolism, transcription factor activity, kinase activity, phosphatase activity, and stress response.

The predicted five KEGG pathways were statistically significant in the transcriptomic profiling (Table S3). For two comparison groups ( $0 \% \mathrm{NaCl}$ vs $20 \% \mathrm{NaCl}$ and $10 \% \mathrm{NaCl}$ vs $20 \% \mathrm{NaCl}$ ), DEGs were enriched in ribosome pathways (45 genes and 51 genes). For the third comparison group $(0 \% \mathrm{NaCl}$ vs $10 \% \mathrm{NaCl})$, DEGs was enriched in glycolysis/gluconeogenesis metabolism (12 genes), pyruvate metabolism (12 genes), and glucagon signaling pathway (4 genes). After transcriptome enrichment analysis, we observed that the genes could be matched to the KEGG database that was mainly concentrated in energy metabolism, carbon and nitrogen metabolism.

\section{Verification by qRT-PCR}

To assess the reliability of our RNA-Seq, 10 DEGs were quantified using qRT-PCR. As shown in Fig. 5, the trend in qRT-PCR expression was in agreement with the RNA-seq expression profile. The results showed similar patterns of mRNA abundance in RNA-seq analysis and qRT-PCR. Therefore, RNA-seq results can reflect the expression of $S$. aureus transcriptome under high salt stress. Transcriptome data can be used for the analysis.

\section{Metabolomic profiling through GC-MS}


In total, 76 endogenous metabolites were identified in S. aureus ZS01. Then, the concentrations of 76 metabolites in these three groups were calculated based on the internal standard peak area (Table S4). Principal component analysis (PCA) is an overall presentation of the distribution of the original data for the samples. As shown in Fig. 6A, the main components of the metabolites were located in different quadrants, indicating clear discrimination among the intracellular metabolome in the three groups. To identify the metabolites affected by salt stress, orthogonal partial least squares discriminant analysis (OPLS-DA) was employed on the metabolic profiles. A total of 15 differential metabolites (DMs) were screened under VIP $>1$ with $p<0.05$ as standard. The $0 \% \mathrm{NaCl}$ vs $10 \% \mathrm{NaCl}$ group screened a total of ten DMs (aminobutanoic acid, glycolic acid, D-erythrofuranose, sebacic acid, xylitol, D-threitol, nhexadecanoic acid, myo-inositol, heptacosane, and undecanedioic acid), the $10 \% \mathrm{NaCl}$ vs $20 \% \mathrm{NaCl}$ group screened a total of six DMs (L-proline, phosphoric acid, butanedioic acid, D-arabinose, D-mannitol, and $\mathrm{n}$-hexadecanoic acid), and the $0 \% \mathrm{NaCl}$ vs $20 \% \mathrm{NaCl}$ group screened a total of six DMs (aminobutanoic acid, L-proline, phosphoric acid, butanedioic acid, D-arabinose, and xylitol) (Table S5).

We subjected the DMs to the pathway analysis to get the overall view of their contributions. The metabolic pathways related to salt stress responses are shown in Fig. 6B, 6C, and 6D. These pathways were mainly involved in inositol phosphate metabolism, sulfur metabolism, and TCA cycle (Table S6). Therefore, the results suggest that initiating defense (sulfur metabolism), signal response (inositol phosphate metabolism), and energy regulation (TCA cycle) are the key response pathways for $S$. aureus ZS01 to salt stress.

\section{Integrated analysis of transcriptomeand metabolome}

We combine transcriptome and metabolome data to gain insight into the regulatory network of $S$. aureus ZS01 under salt stress conditions. The transcriptome finally identified 81 related genes, and performed Spearman correlation analysis with $15 \mathrm{DMs}$. Taking a $p \leq 0.05$ as the threshold, paired regulatory relationships were plotted (Fig. 7). A total of 80 nodes that were connected in the network with 236 edges are displayed in the visualization of Cytoscape. According to the edge greater than ten genes (antB, fnbA, gale, hisD, hisG, lysC, $m n h D$, proP, sdrC_D_E and serA) for salinity stress response were obtained. Furthermore, all of them were present in Pattern III (decrease/decrease) expression change. Overall, the integrated multiomics analysis identified hub genes that were potentially linked to salt stress. They deserve further attention and in-depth functional study and validation for applications.

\section{Discussion}

SFP is one of the pathogenic factors in the process of aquatic production and preservation under high salt conditions all over the world (Hennekinne et al., 2012). The salt-tolerant $S$. aureus is the main factor that plagues the quality and safety of aquatic products. However, the current mechanism of $S$. aureus adaptation to salt stress is still insufficient. Therefore, this study used a variety of omics methods to clarify this mechanism in a comprehensive manner. We observed that $S$. aureus suffered a large difference in response to salinity stress. Phenotypic and physiological adaptation to environmental 
conditions is driven by changes in gene regulation, metabolite accumulation, and cell signal transduction. We determined that biofilm formation, signal response, transcription factors, and energy metabolism played important roles in the salt stress defensive system of $S$. aureus.

\section{Biofilm formation}

Extracellular polymeric substances (EPS) contributed to biofilm stability and adhesion properties. Capsular polysaccharide (CPS) is the main resistant component of EPS and a major virulence factor in $S$. aureus (Lee et al., 1992; Lee et al., 1994). With the help of biofilms, bacteria exhibit more resistance to salinity stress. The change pattern of biofilm formation after salt stress is the same as the expression change Pattern II (increase/decrease) of transcriptome. In Pattern II, we found that CPS biosynthesis protein genes, such as cap5A, cap5B, cap8C, capD, cap8F, and cap5D, were candidate target genes for direct regulation of salt stress. Microbial biofilms can be used as a "protective suit" to protect microorganisms from extreme temperatures, ultraviolet radiation, extreme $\mathrm{pH}$, high salinity, etc. (Yin et al., 2019). The natural ability of microorganisms to deal with harsh environments is attributed to their genetic diversity and physiological adaptability. Previous studies have indicated that bacterial EPS can help alleviate salt stress by reducing the content of sodium (Banerjee et al., 2019). So, we believe that the formation of biofilm and the production of EPS are important strategies for salt-tolerant $S$. aureus to assist in metabolism in response to salt stress. Enhanced production of EPS is the significant protection mechanism of periphytic biofilms against high concentrations of $\mathrm{NaCl}$. At the same time, according to biofilm phenotype changes and differential gene expression, it can be found that the critical point of $10 \%$ $\mathrm{NaCl}$ is a relatively special state. These results can be used to explain the different mechanisms in $S$. aureus for tolerating ordinary high-salt and extreme high-salt.

\section{Signal response}

DM myo-inositol (VIP $=1.31)$ was downregulated significantly by 0.79 -fold in the $10 \% \mathrm{NaCl}$ group $(p<$ 0.05) compared to the control group. At the same time, inositol phosphate metabolism has the greatest effect on this group. D-Glucose-6-phosphate is the only source of inositol ring (Raboy et al., 1996). In this study, the downregulation of fructose-1,6-bisphosphatase gene $f b p 3$ resulted in the decrease of glucose 6 phosphate content, which was the main reason for the downregulation of inositol content. Shao et al. proved that phosphoinositide has a synergistic effect with carbohydrate transporters, and it is closely related to ion transport (Shao et al., 2016). It can be inferred that the reduction of endogenous glucose was the main reason for the downregulation of S. aureus ZS01 inositol phosphate metabolism. The functions of phosphoinositide are diverse, involving processes such as stress resistance, signal response, conversion between secondary metabolites, DNA repair, and RNA transport (Hanakahi et al., 2000; Bolger et al., 2008). Inositol phosphate metabolism is complicated. It involves the conversion of various inositol phosphate molecules, which perform various functions in metabolic pathways (Fig. 8). Therefore, in this study, we speculated that $S$. aureus ZS01 reduced the conversion of functional molecules, and downregulated inositol phosphate metabolism is one of its strategies to adapt to salt stress. 
Studies have shown that osmotic stress can induce the production of a variety of stress proteins, which have the functions, such as cell self-protection, nucleic acid repair, abnormal protein degradation, regulation of intracellular, and extracellular osmotic pressure (Kiran and Naomi, 2009; Vilhelmsson and Miller, 2002). General stress protein gene $d p s$ and universal stress protein gene uspA-7and uspA-2 were upregulated significantly in the $10 \% \mathrm{NaCl}$ group $(p<0.01)$, while downregulated significantly in the $20 \%$ $\mathrm{NaCl}$ group $(p<0.01)$. In $S$. aureus, both general stress response and universal stress response are widely conserved responses allowing bacteria to cope with a multitude of stressful conditions. Cold shock protein gene $(\operatorname{csp} A, \operatorname{csp} C)$ and heat shock protein gene $(g r p E)$ showed significant up-down changes during the process of salt stress enhancement. Our results indicate that these DEGs not only respond to high and low temperature stress but also play an important role in resistance to osmotic stress. They may act as a full range of firefighters and respond to various extreme environmental changes.

\section{Transcription factors}

A higher abundance of transcription factors (TFs) as key regulators of transcription is important in $S$. aureus responses to salinity stress. Transcriptome analysis revealed that members of $>30$ TF families are responsive to salinity stress in candidate genes. Through the analysis of TF genes, the MarR family held the largest number, which reached 9 . Evidence has shown that MarR family transcription regulators are sensors that sense changes in the environment, can bind small molecule compounds, and quickly start adaptive regulation of transcription levels, including controlling the production of virulence factors, responses to antibiotics and oxidative stress, and catabolism of aromatic compounds in the environment (Wei et al., 2007; Deochand and Grove, 2017)[24-26]. The GntR family also dominates in number. Previous studies have shown that GntR family TF combines with metabolites through allosteric effects, regulates the expression of genes, such as metabolism, drug resistance, and virulence, and responds quickly to environmental signals (Kunin and Rudy, 1991; Rigali et al., 2002). These results indicate that $S$. aureus ZS01 may be resistant to external stress by regulating the expression of certain stress tolerance genes or drug resistance genes. They turn on different regulatory systems through transcriptional regulation, so that cells can adapt to changes in the environment.

\section{Energy metabolism}

Transcriptome enrichment analysis results show that critical pathways are mainly concentrated in energy metabolism, carbon and nitrogen metabolism. Metabolome enrichment analysis results show that critical pathways are mainly concentrated in several energy metabolic pathways such as fatty acid biosynthesis, fatty acid degradation, TCA cycle, fructose and mannose metabolism. From the above results, we conclude that the regulation of energy metabolism pathways is an important strategy for $S$. aureus ZS01 to cope with salt stress.

Fructose-1,6-bisphosphatase fbp3 is one of the key rate-limiting enzymes in gluconeogenesis. It plays a major role in regulating changes in glucose content and plays an important role in sugar metabolism (Brown et al., 2009). In this study, fbp3 was continuously downregulated with the increase of salt stress. Eventually, it leaded to downregulation of the gluconeogenesis pathway. In the process of salt stress, $S$. 
aureus ZS01 reduced energy consumption by reducing the production of endogenous glucose. In the transcriptome, the pyruvate metabolism pathway fumarate hydratase E4.2.1.2 and formate acetyltransferase E2.3.1.54 were upregulated, leading to the upregulation of succinate content (Fig. 8). It can be found that high salt stress can promote the accumulation of succinate. The accumulation of this substance can reduce the sensitivity of the bacteria to salt stress, thereby improving the ability of the bacteria to respond to salt stress. This result has been verified in the metabolome, the relative content of DM succinate was continuously upregulated, and it participated in the TCA cycle.

ATP is the key energy substance that sustains life activities in the process of microbial metabolism. $\mathrm{NADH}$ or $\mathrm{FADH}_{2}$ reacts with oxidized phosphoric acid via electron transfer to produce a large amount of ATP. In the two comparison groups where TCA is active, according to the transcriptome data, there are 3 common differentially expressed genes in the electron transport chain, ssuE (NADH-dependent FMN reductase), $n d h F$ (NADH dehydrogenase subunit 5) and $s d h C$ (succinate dehydrogenase cytochrome b558 subunit) expression levels are downregulated. According to the changes in the expression levels of genes related to the electron transport chain, it is speculated that high salt has an inhibitory effect on the electron transport chain of $S$. aureus ZS01. The utilization of ATP also depends on the enzymes of the citrate cycle. As three key rate-limiting enzymes in the TCA metabolic pathway, the expression levels of gltA (citrate synthase), icd (isocitrate dehydrogenase) and sucA (2-oxoglutarate dehydrogenase E1 component) are downregulated. Downregulation of TCA cycle related enzymes in S. aureus ZS01 provides evidence for energy regulation to maintain survivable growth under salinity. The TCA overall show a low expression trend to maintain cell proliferation under high salt stress. As the salt concentration increases, the transcription of the gluconeogenesis decrease, which reduces the intracellular transport efficiency of glucose. The downregulation of these two energy metabolism pathways indicate that the cells are in a state of low energy metabolism similar to self-protection under high salt stress. In this respect, our results are consistent with previous study (Ming et al., 2019). Previous studies have shown that self-protection mechanisms can protect cells from hypertonic environments, reduce unnecessary energy loss, and help them focus on survival (Lee, 2013). Although stronger stress can hinder cell development and biomass, the life cycle is maintained, suggesting an adapted metabolism able to counteract this stress (Arbelet-Bonnin et al., 2020). We conclude that low energy metabolism was an important way to self-protect and overcome high salt stress for $S$. aureus ZS01.

\section{Conclusion}

S. aureus has an extraordinary ability to deal with a wide range of salinity changes. The exhaustive profiling of genes, metabolites, and pathways revealed important information regarding the regulatory networks underlying bacteria responses to salt stress. In this study, we confirmed that biofilm formation, signal response, transcription factors, and energy metabolism played important roles in the salt stress defensive system of $S$. aureus ZS01. Low energy metabolism is the most important self-protection strategy for $S$. aureus to cope with salinity stress. We can selectively screen potential targets for salt tolerance and pathogenicity of $S$. aureus and suppress the pathogenicity and toxicity, helping us to stop 
SFB infections. Based on the analysis of omics data, this study provides new insight into selected potential targets for salt tolerance and pathogenicity of $S$. aureus in aquatic production and preservation.

\section{Declarations}

\section{Funding}

This work was supported by the National Key R\&D Program of China (2019YFD0900101), the National Key R\&D Program of China (Grant 2017YFC1404505), the K.C. Wong Magna Fund in Ningbo University and Scientific Research Project of Jilin Provincial Department of Education (JJKH20210531KJ).

\section{Conflict of interest}

The authors declare that they have no competing interests.

\section{Availability of data}

The raw sequencing data were deposited at the NCBI Sequence Read Archive (https://www.ncbi.nlm.nih.gov/sra) under Bioproject No. PRJNA704096.

\section{Authors' contributions}

RXW, XRS, CYL, and JZ conceived and designed the experiments. YF, WZY, DZG, and JFF carried out the experiments and data analysis. RXW, XRS, YF, and CYL were involved in drafting the manuscript. All the authors read and approved the final manuscript.

\section{References}

1. Ai X, Gao F, Yao S, Liang B, Mai J, Xiong Z, Chen X, Liang Z, Yang H, Ou Z, Gong S, Long Y, Zhou Z (2020). Prevalence, characterization, and drug resistance of Staphylococcus aureus in feces from pediatric patients in Guangzhou, China. Frontiers in Medicine 7: 127.

2. Alibayov B, Zdeňková K, Purkrtová S, Demnerová K, Karpíšková R. (2014). Detection of some phenotypic and genotypic characteristics of Staphylococcus aureus isolated from food items in the Czech Republic. Annals of Microbiology 64: 1587-1596.

3. Angelidis AS, Smith GM (2003). Three transporters mediate uptake of glycine betaine and carnitine by Listeria monocytogenes in response to hyperosmotic stress. Applied \& Environmental Microbiology 69: 1013-1022.

4. Arbelet BD, Blasselle C, Rose PE, Redwan M, Ponnaiah M, Laurenti P, Meimoun P, Gilard F, Gakière B, Mancuso S, El-Maarouf BH, Bouteau F (2020). Metabolism regulation during salt exposure in the halophyte Cakile maritima. Environmental \& Experimental Botany 177: http://www.ncbi.nlm.nih.gov/pubmed/104075. 
5. Argudín MÁ, Mendoza MC, Rodicio MR (2010). Food poisoning and Staphylococcus aureus enterotoxins. Toxins 2: 1751-1773.

6. Banerjee A, Sarkar S, Cuadros OS, Bandopadhyay R (2019) Exopolysaccharides and Biofilms in Mitigating Salinity Stress: The Biotechnological Potential of Halophilic and Soil-Inhabiting PGPR Microorganisms. Soil Biology 56: 133-153.

7. Beckingsale, Thomas (2008). Staphylococcus aureus: Salt Stress, Cell Wall Deficiency, and Biofílm Formation. Durham theses: 17-64.

8. Boch J, Kempf B, Bremer E (1994). Osmoregulation in Bacillus subtilis: synthesis of the osmoprotectant glycine betaine from exogenously provided choline. Journal of Bacteriology 176: 5364-5371.

9. Bolger TA, Folkmann AW, Tran EJ, Wente SR (2008). The mRNA Export Factor Gle1 and Inositol Hexakisphosphate Regulate Distinct Stages of Translation. Cell 134: 624-633.

10. Brown G, Singer A, Lunin VV, Proudfoot M, Skarina T, Flick R, Kochinyan S, Sanishvili R, Joachimiak A, Edwards AM, Alexei S, Alexander F, Yakunin AK (2009). Structural and Biochemical Characterization of the Type II Fructose-1,6-bisphosphatase GIpX from Escherichia coli *. Journal of Biological Chemistry 284: 3784-3792.

11. Cebrián G, Arroyo C, Condón S, Manas P (2015). Osmotolerance provided by the alternative sigma factors $\sigma(\mathrm{B})$ and rpoS to Staphylococcus aureus and Escherichia coli is solute dependent and does not result in an increased growth fitness in $\mathrm{NaCl}$ containing media. International Journal of Food Microbiology 214: 83-90.

12. Chong J, Wishart DS, Xia J (2019). Using MetaboAnalyst 4.0 for comprehensive and integrative metabolomics data analysis. Current Protocols in Bioinformatics 68: e86.

13. Cole T, Brian AW, Geo P, Ali M, Gordon K, Marijke VB, Steven LS, Barbara JW, Lior P (2010). Transcript assembly and quantification by RNA-Seq reveals unannotated transcripts and isoform switching during cell differentiation. Nature Biotechnology 28: 511-515.

14. Deng W, Wang Y, Liu Z, Cheng H, Xue Y (2014). Heml: A toolkit for illustrating heatmaps. PLOS ONE 9: e111988.

15. Deochand DK, Grove A (2017). MarR family transcription factors: dynamic variations on a common scaffold. Critical Reviews in Biochemistry \& Molecular Biology 52: 595-613.

16. Fischer A, Francois P, Holtfreter S, Broeker B, Schrenzel J (2009). Development and evaluation of a rapid strategy to determine enterotoxin gene content in Staphylococcus aureus. Journal of Microbiological Methods 77: 184-190.

17. Fuentes A, Fernández-segovia I, Barat JM, Serra JA (2010). Physicochemical characterization of some smoked and marinated fish products. Journal of Food Processing \& Preservation 34: 83-103.

18. Gansauge MT, Meyer M. (2013). Single-stranded DNA library preparation for the sequencing of ancient or damaged DNA. Nature Protocols 8: 737-748.

19. Graham JE, Wilkinson BJ (1992). Staphylococcus aureus osmoregulation: roles for choline, glycine betaine, proline, and taurine. Journal of Bacteriology 174: 2711-2716. 
20. Gruszczyk J, Fleurie A, Olivares IV, Béchet E, Zanella Cl, Moréra S, Meyer P, Pompidor G, Kahn R, Grangeasse C, Nessler S (2011). Structure analysis of the Staphylococcus aureus UDP-N-acetylmannosamine dehydrogenase Cap50 involved in capsular polysaccharide biosynthesis. Journal of Biological Chemistry 286: 17112-17121.

21. Guan XL, Zhao YJ, Liu X, Shang B, Xing F, Zhou L (2019). The bZIP transcription factor Afap1 mediates the oxidative stress response and aflatoxin biosynthesis in Aspergillus flavus. Revista Argentina de Microbiologia 51: 292-301.

22. Hanakahi LA, Bartlet JM, Chappell C, Pappin D, West SC (2000). Binding of Inositol Phosphate to DNA-PK and Stimulation of Double-Strand Break Repair. Cell 102: 721-729.

23. Hashim Z, Mukai Y, Bamba T, Fukusaki E (2014). Metabolic profiling of retrograde pathway transcription factors $\mathrm{rtg} 1$ and rtg3 knockout yeast. Metabolites 4: 580-598.

24. Hennekinne JA, De Buyser ML, Dragacci S (2012). Staphylococcus aureus and its food poisoning toxins: characterization and outbreak investigation. FEMS Microbiology Reviews 36: 815-836.

25. Islam N, Ross JM, Marten MR (2015). Proteome Analyses of Staphylococcus aureus Biofilm at Elevated Levels of $\mathrm{NaCl}$. Clinical Microbiology 4: 219.

26. Kiran MD, Naomi B (2009). TRAP Plays a Role in Stress Response in Staphylococcus aureus. International Journal of Artificial Organs 32: 592-599.

27. Kumari A, Parida AK (2018). Metabolomics and network analysis reveal the potential metabolites and biological pathways involved in salinity tolerance of the halophyte Salvadora persica. Environmental and Experimental Botany 148: 85-99.

28. Kunin CM, Rudy J (1991). Effect of NaCl-induced osmotic stress on intracellular concentrations of glycine betaine and potassium in Escherichia coli, Enterococcus faecalis, and staphylococci. The Journal of Laboratory and Clinical Medicine 118: 217-224.

29. Lee CY (1992). Cloning of genes affecting capsule expression in Staphylococcus aureus strain M. Molecular Microbiology 6: 1515-1522.

30. Lee JC, Xu S, Albus A, Livolsi PJ (1994). Genetic analysis of type 5 capsular polysaccharide expression by Staphylococcus aureus. Journal of Bacteriology 176: 4883-4889.

31. Loir YL, Baron F, Gautier M (2003). Staphylococcus aureus and food poisoning. Genetics \& Molecular Research 2: 63-76.

32. Ming TH, Geng L, Feng Y, Lu C, Zhou J, Li Y, Zhang D, He S, Li Y, Cheong L, Su X (2019). iTRAQ-based quantitative proteomic profiling of Staphylococcus aureus under different osmotic stress conditions. Frontiers in Microbiology 10: 1082.

33. Ming TH, Han JJ, Li YY, Lu CY, Qiu D, Li Y, Zhou J, Su XR. (2018). A metabolomics and proteomics study of the Lactobacillus plantarum in the grass carp fermentation. BMC Microbiology 18: 216.

34. Nagayama K, Fujita K, Takashima Y, Ardin AC, Ooshima T, Matsumoto NM (2014). Role of ABC transporter proteins in stress responses of Streptococcus mutans. Oral Health \& Dental Management 13: 359-365. 
35. Oshima T, Aiba H, Masuda Y, Kanaya S, Sugiura M, Wanner BL, Mori H, Mizuno T (2002). Transcriptome analysis of all two-component regulatory system mutants of Escherichia coli K-12. Molecular Microbiology 46: 281-291.

36. Price WA, Poon CK, Benson MA, Eidem TT, Roux CM, Boyd JM, Dunman PM, Torres VJ, Krulwich, TA (2013). Transcriptional profiling of Staphylococcus aureus During growth in $2 \mathrm{M} \mathrm{NaCl}$ leads to clarification of physiological roles for Kdp and $\mathrm{Ktr}^{+}$uptake systems. mBio 4: e00407-00413.

37. Raboy V, Gerbasi P (1996). Genetics of myo-inositol phosphate synthesis and accumulation. Subcellular biochemistry 26: 257-285.

38. Ran S, Liu B, Jiang W, Sun Z, Liang J. (2015). Transcriptome analysis of Enterococcus faecalis in response to alkaline stress. Frontiers in Microbiology 6: 795.

39. Ran S, Liu B, Jiang W, Sun Z, Liang J (2015b). Transcriptome analysis of Enterococcus faecalis in response to alkaline stress. Frontiers in Microbiology 6: 795.

40. Rigali S, Derouaux A, Giannotta F, Dusart J. (2002). Subdivision of the helix-turn-helix GntR family of bacterial regulators in the FadR, HutC, MocR, and YtrA subfamilies. Journal of Biological Chemistry 277: 12507-12515.

41. Rodriguez MC, Riesco PF, Garcia FC, Alonso CC, Capita R (2019). Susceptibility of Listeria monocytogenes planktonic cultures and biofilms to sodium hypochlorite and benzalkonium chloride. Food Microbiology 82: 533-540.

42. Schuster CF, Bellows LE, Tosi T, Campeotto I, Corrigan RM, Freemont P, Gründling A (2016). The second messenger c-di-AMP inhibits the osmolyte uptake system OpuC in Staphylococcus aureus. Science Signaling 9: ra81.

43. Sergelidis D, Abrahim A, Papadopoulos T, Soultos N, Martziou E, Koulourida V, Govaris A, Pexara A, Zdragas A, Papa A (2014). Isolation of methicillin-resistant Staphylococcus spp. from ready-to-eat fish products. Letters in Applied Microbiology 59: 500-506.

44. Zhang S, Yang W, Zhao Q, Zhou X, Jiang L, Ma S, Liu X, Li Y, Zhang C, Fan Y, Chen R. Analysis of weighted co-regulatory networks in maize provides insights into new genes and regulatory mechanisms related to inositol phosphate metabolism. BMC Genomics 17: 129.

45. Vijaranakul U, Nadakavukaren MJ, De Jonge BL, Wilkinson BJ, Jayaswal RK (1995). Increased cell size and shortened peptidoglycan interpeptide bridge of $\mathrm{NaCl}$-stressed Staphylococcus aureus and their reversal by glycine betaine. Journal of Bacteriology 177: 5116-5121.

46. Vilhelmsson O, Miller KJ (2002). Synthesis of pyruvate dehydrogenase in Staphylococcus aureus is stimulated by osmotic stress. Applied \& Environmental Microbiology 68: 2353-2358.

47. Wei K, Tang DJ, He YQ, Feng JX, Jiang BL, Lu GT, Chen B, Tang JL (2007). hpaR, a putative marR family transcriptional regulator, is positively controlled by $\mathrm{HrpG}$ and $\mathrm{HrpX}$ and involved in the pathogenesis, hypersensitive response, and extracellular protease production of Xanthomonas campestris pathovar campestris. Journal of Bacteriology 189: 2055-2062.

48. Wong TW, Liao SZ, Ko WC, Wu CJ, Wu SB, Chuang YC, Huang IH (2019). Indocyanine green-mediated photodynamic therapy reduces methicillin-resistant Staphylococcus aureus Drug resistance. Journal 
of Clinical Medicine 8: 411.

49. Xin W, Zhang L, Zhang W, Gao J, Yi J, Zhen X, Li Z, Zhao Y, Peng C, Zhao C (2019). An integrated analysis of the rice transcriptome and metabolome reveals differential regulation of carbon and nitrogen metabolism in response to nitrogen availability. International Journal of Molecular Sciences 20: 2349 .

50. Xu Z, Xie J, Soteyome T, Peters BM, Shirtliff ME, Liu J, Harro JM (2019). Polymicrobial interaction and biofilms between Staphylococcus aureus and Pseudomonas aeruginosa: an underestimated concern in food safety. Current Opinion in Food Science 26: 57-64.

51. Yin W, Wang Y, Liu L, He J (2019). Biofilms: The Microbial "Protective Clothing" in Extreme Environments. International journal of molecular sciences 20: 3423.

52. Zhang X, Wen H, Wang H, Ren Y, Zhao J, Li Y (2017). RNA-Seq analysis of salinity stress-responsive transcriptome in the liver of spotted sea bass (Lateolabrax maculatus). PLOS ONE 12: e0173238.

53. Zhao X, Liu Z, Liu Z, Meng R, Shi C, Chen X, Bu X, Guo N (2018). Phenotype and RNA-seq-Based transcriptome profiling of Staphylococcus aureus biofilms in response to tea tree oil. Microbial Pathogenesis 123: 304-313.

\section{Figures}
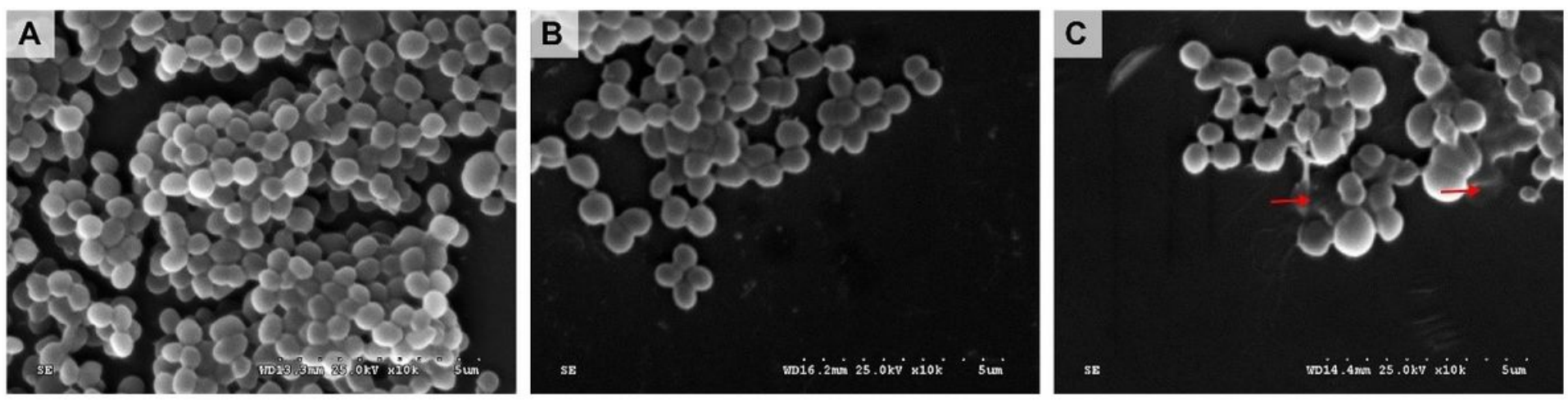

Figure 1

Morphology of S. aureusZS01 under SEM. (A) 0\% NaCl group; (B) 10\% NaCl group; (C) $20 \% \mathrm{NaCl}$ group. SEM images of $\mathrm{NaCl}$ treated and untreated S. aureus ZS01cells. S. aureus ZS01 was incubated for 48 hours in a broth medium supplemented with $0 \%, 10 \%$ and $20 \% \mathrm{NaCl}$, respectively. $\times 10 \mathrm{~K}$-fold. Red solid arrows have indicated major structural changes.
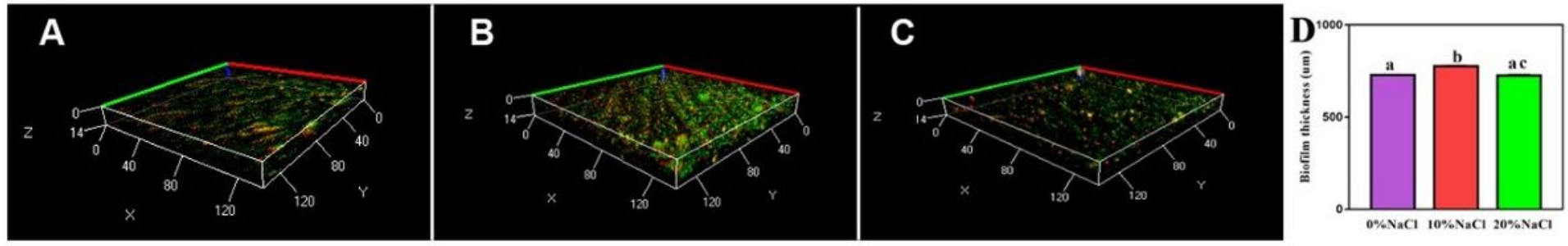
Figure 2

CLSM images of S. aureus ZS01 biofilm formation changes. (A) $0 \% \mathrm{NaCl}$ group; (B) $10 \% \mathrm{NaCl}$ group; (C) $20 \% \mathrm{NaCl}$ group; (D) The thickness of biofilm. S. aureus ZS01 was incubated for 48 hours in a broth medium supplemented with $0 \%, 10 \%$ and $20 \% \mathrm{NaCl}$, respectively. The concentration of the collected bacterial solution is $5 \times 106 \mathrm{CFU} / \mathrm{mL}$. Biofilm was stained with FITC-ConA $\left(4^{\circ} \mathrm{C}, 30 \mathrm{~min}\right)$ and $\mathrm{PI}\left(4^{\circ} \mathrm{C}\right.$, $15 \mathrm{~min}$ ), then imaged. $\lambda$ ex $=488 \mathrm{~nm}, \lambda \mathrm{em}=520 \mathrm{~nm}$ was used for FITC-ConA detection (green channel), $\lambda e x=$ $543 \mathrm{~nm}, \lambda \mathrm{em}=572 \mathrm{~nm}$ was used for PI detection (red channel). Three fields of view are randomly selected for each specimen and scanned layer by layer along the $Z$ axis. CLSM images were captured using a Zeiss LSM880 confocal laser scanning microscope (Zeiss LSM880, Germany) with $\times 40$ objective lens. Z stack represents the thickness of biofilm.

A

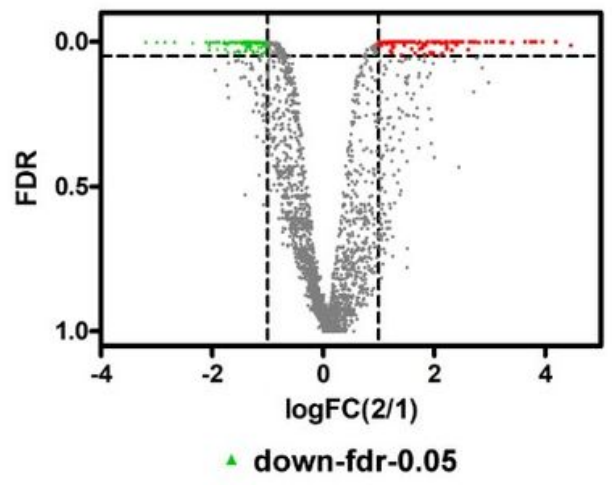

B

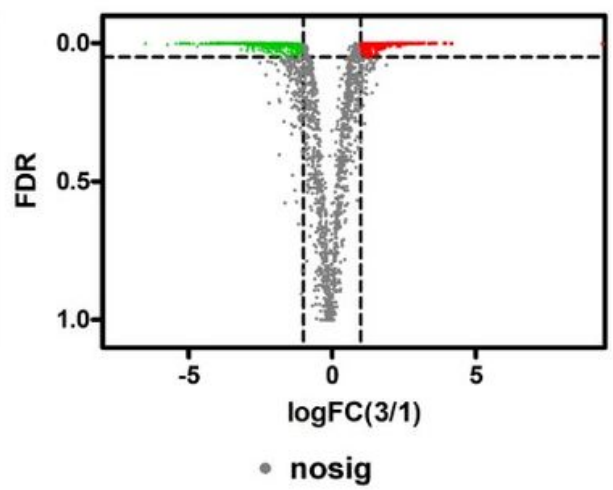

C

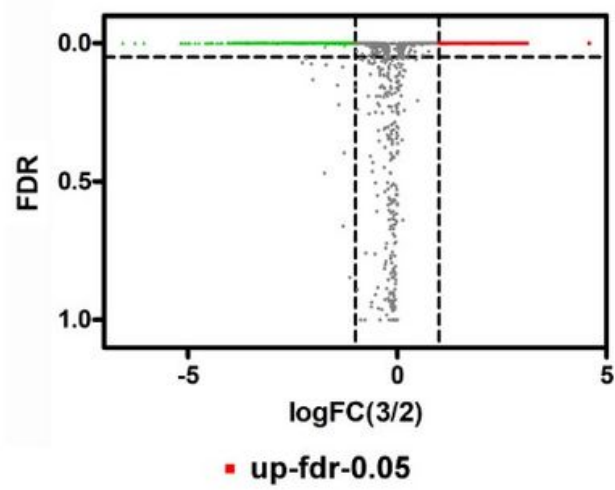

Figure 3

Volcano plot of the genes identified in S. aureus ZS01 treated with different concentrations of $\mathrm{NaCl}$. (A) $0 \% \mathrm{NaCl}$ vs $10 \% \mathrm{NaCl}$; (B) $0 \% \mathrm{NaCl}$ vs $20 \% \mathrm{NaCl}$; (C) $10 \% \mathrm{NaCl}$ vs $20 \% \mathrm{NaCl}$. Each point represents a gene, and the red and green areas represents upregulated (Log2FC $\geq 1$ and FDR $\leq 0.05)$ and downregulated (Log2FC $\leq-1$ and FDR $\leq 0.05)$ genes, respectively. 


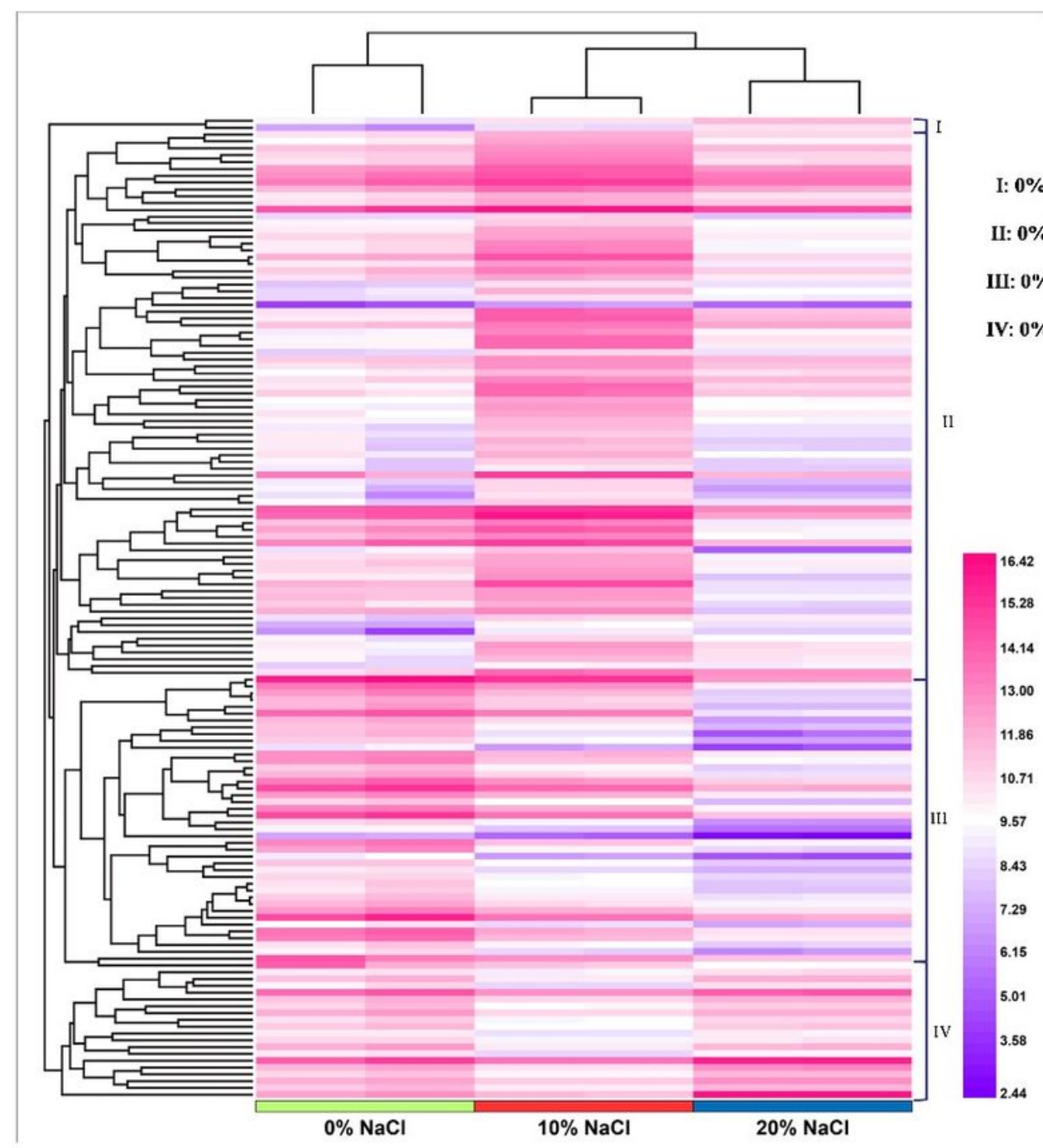

Figure 4

Functional clustering analysis of the DEGs with increasing $\mathrm{NaCl}$ concentrations. Pattern I (increase/increase): genes that were stepwise upregulated with increasing $\mathrm{NaCl}$ concentrations; Pattern II (increase/decrease): genes that reached their highest expression levels in the $10 \% \mathrm{NaCl}$ group; Pattern III (decrease/decrease): genes that were stepwise downregulated with increasing $\mathrm{NaCl}$ concentrations and Pattern IV (decrease/increase): genes that reached their lowest expression levels in the $10 \% \mathrm{NaCl}$ group. 


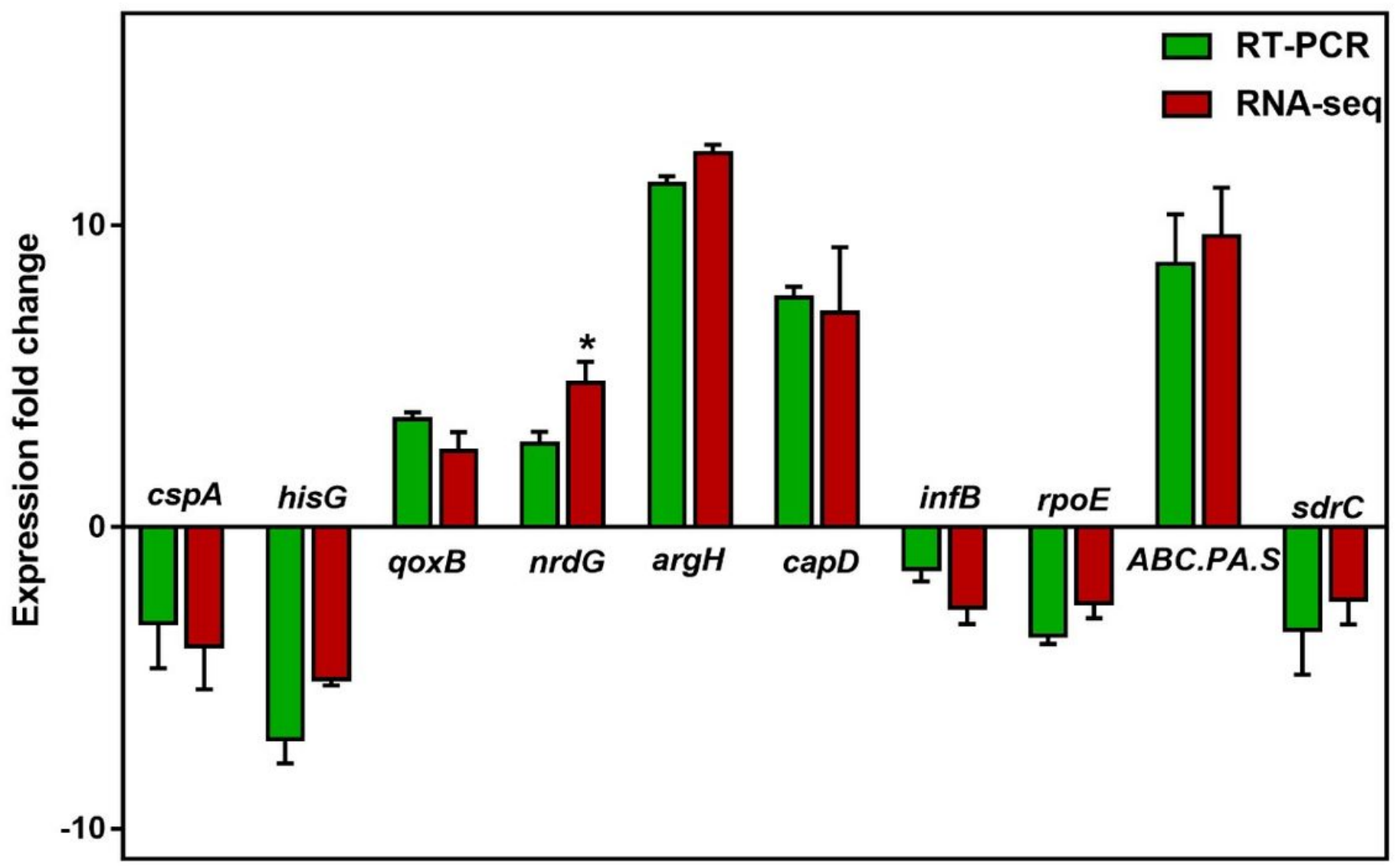

Figure 5

Experimental validation of DEGs by qRT-PCR assays. Candidate genes mainly included cold shock protein gene (cspA), ATP phosphoribosyltransferase gene (hisG), quinol oxidase subunit 1 gene (qoxB), anaerobic ribonucleotide reductase small subunit gene (nrdG), argininosuccinate lyase $(\arg \mathrm{H})$, capsular polysaccharide synthesis enzyme gene (capD), translation initiation factor (IF)-2 gene (infB), DNAdirected RNA polymerase subunit delta gene (rpoE), glutamate $A B C$ transporter permease gene (ABC.PA.S), serine-aspartate repeat protein $\mathrm{F}$ gene ( $\mathrm{sdrC}$ ). The expression of the fold change in the $\mathrm{Y}$-axis indicates the ratios of DEGs (red bar) or mRNA level (green bar). 


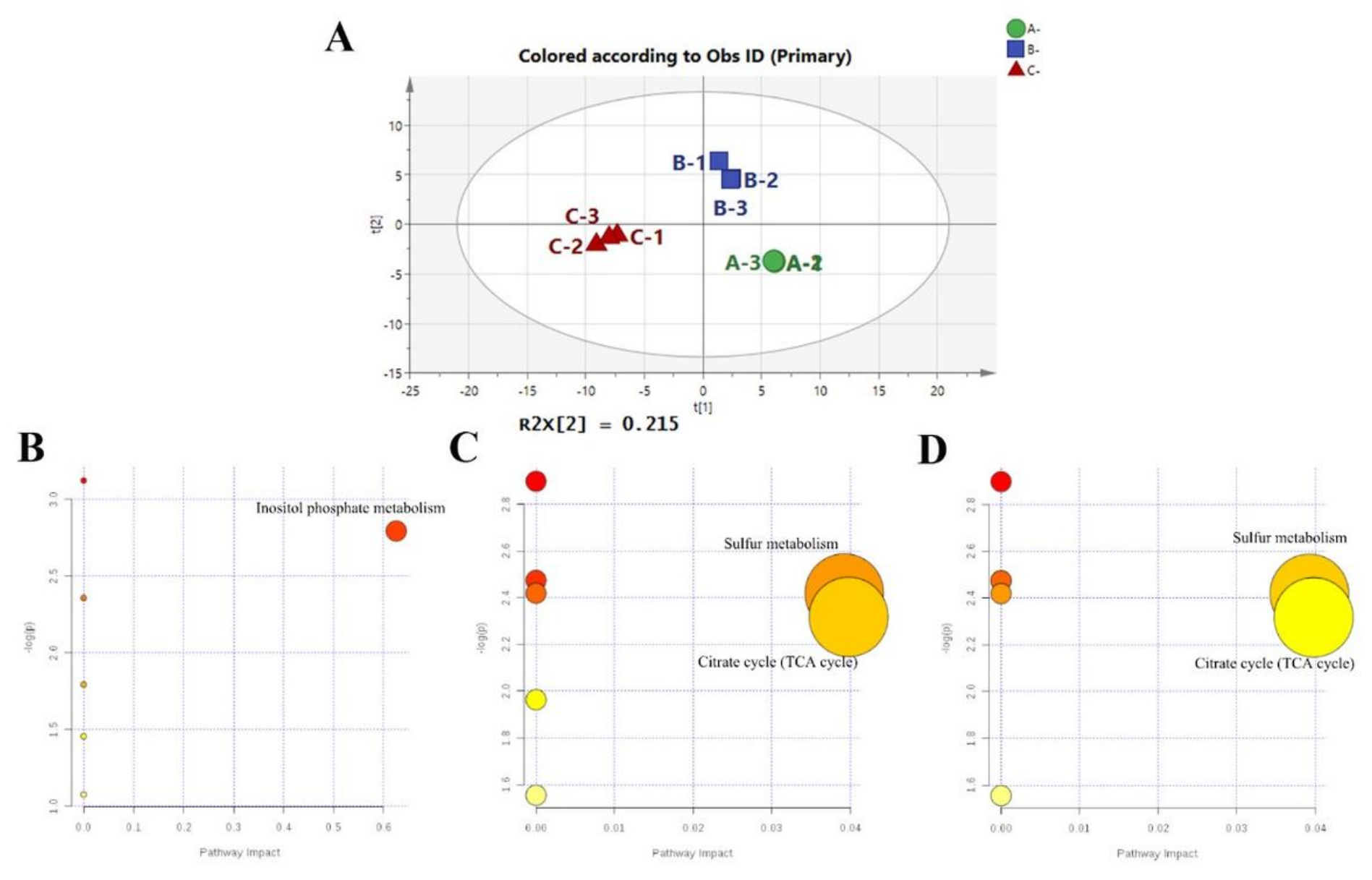

\section{Figure 6}

Score plot of principal components analysis (PCA) and pathway analysis for the biomarkers. (A) Score plot of PCA of the $0 \% \mathrm{NaCl}$ group (green circle), $10 \% \mathrm{NaCl}$ (blue square) and $20 \% \mathrm{NaCl}$ group (red triangle). The t[1] and t[2] values represent the scores of each metabolite sample in the principal components 1 and 2, respectively. The ellipse indicates the 95\% confidence border. Separation among different samples can be seen. (B) Bubble plot of significant metabolic pathways ( $0 \% \mathrm{NaCl}$ vs $10 \% \mathrm{NaCl}$ ); (C) Bubble plot of significant metabolic pathways (10\% NaCl vs $20 \% \mathrm{NaCl}$ ); (D) Bubble plot of significant metabolic pathways $(0 \% \mathrm{NaCl}$ vs $20 \% \mathrm{NaCl})$. According to the path enrichment analysis and pathway impact values, the matched pathway is displayed. Circles represent matched metabolic pathways, which were retrieved from KEGG. The color intensity indicates the significance of the pathway, while size indicates the pathway impact score (the centrality of its involved metabolites). The bigger the value of $\log 10(P)$, the more this pathway is disturbed. The bigger the value of impact, the more this pathway is disturbed. 


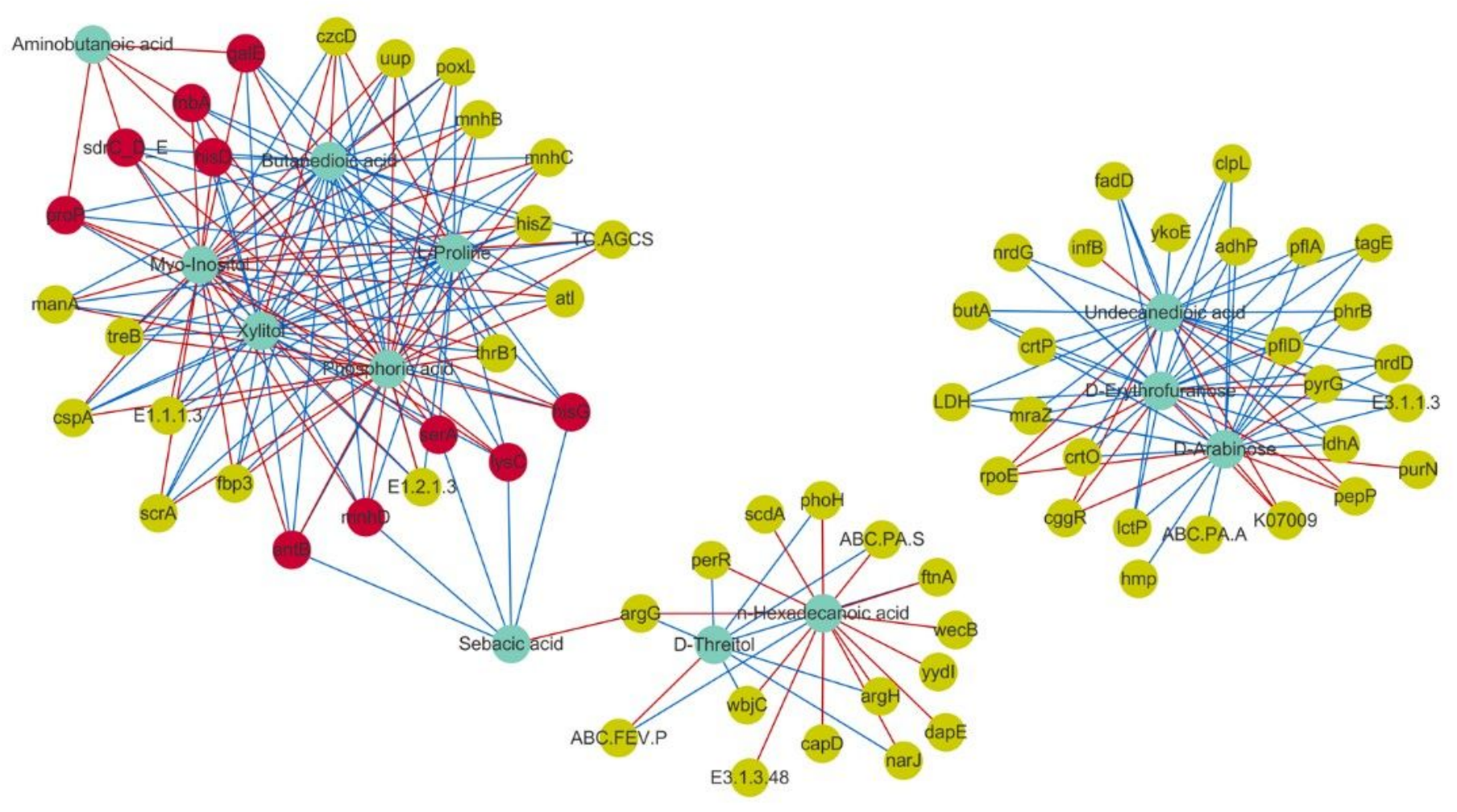

Figure 7

Integrated Analysis of Transcriptome and Metabolome. The Spearman correlation network reveals the regulatory mechanisms of the salinity stress response mechanisms. Results of the correlation analysis between key candidate genes and discriminant metabolites. Different colors of nodes represent metabolites (blue), genes (yellow), and hub genes (red). Positive correlations are indicated by a red line, and negative correlations are indicated by a green line. The thickness of the line represents the magnitude of the correlation coefficient. 


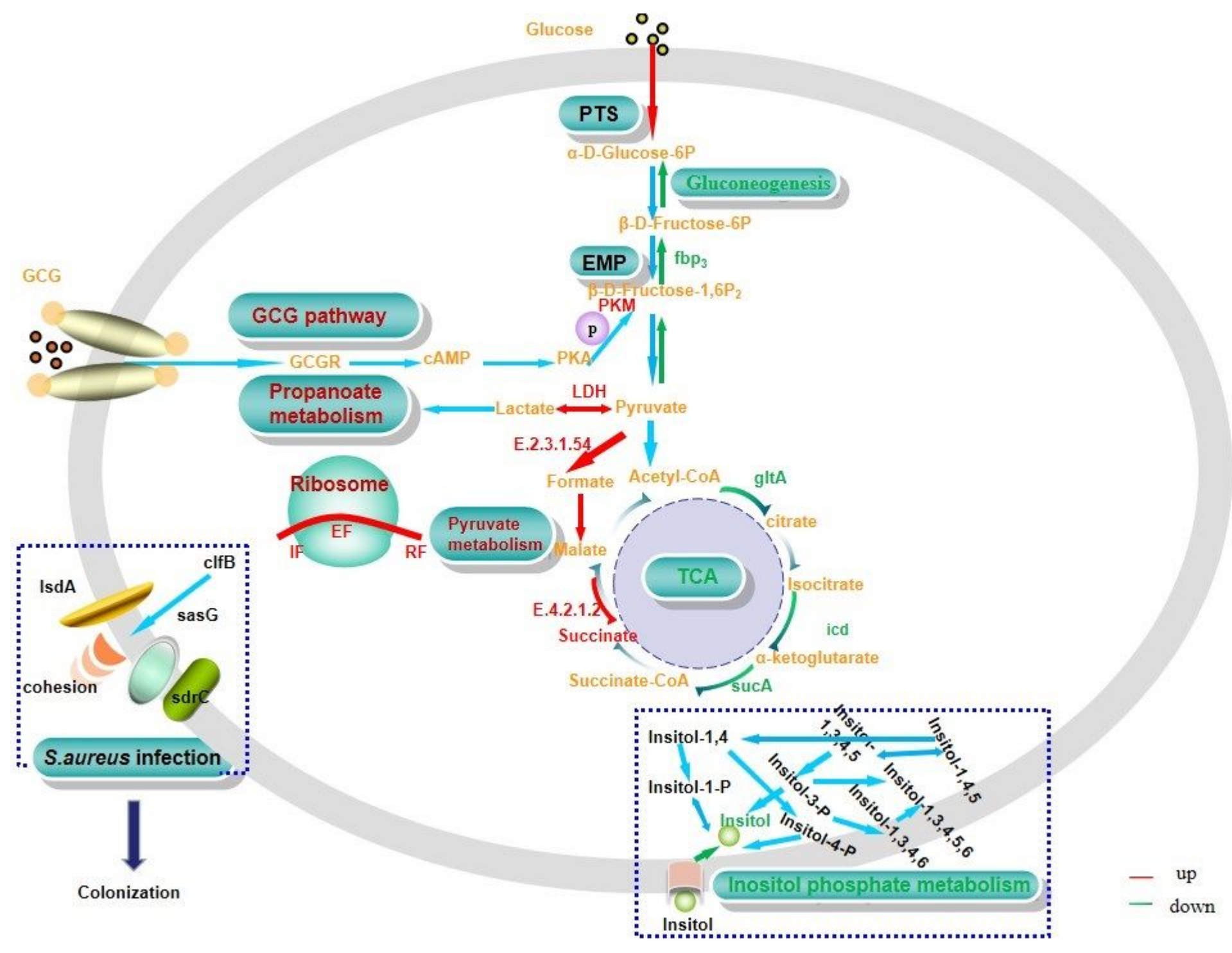

Figure 8

Relevant metabolic pathways in S. aureus ZS01. Red font indicates upregulated genes, and green font indicates downregulated genes. Matched genes include fructose-1,6-bisphosphatase (fbp3), fumarate hydratase (E4.2.1.2), formate acetyltransferase (E2.3.1.54), citrate synthase (gltA), isocitrate dehydrogenase (icd) and 2-oxoglutarate dehydrogenase E1 component (sucA). IF means initiation factor, $\mathrm{EF}$ means elongation factor, RF means release factor.

\section{Supplementary Files}

This is a list of supplementary files associated with this preprint. Click to download.

- SupplementaryMaterial.rar 